\title{
Research on Fatigue Life of Lifting Equipment Based on Nonlinear Cumulative Damage Theory
}

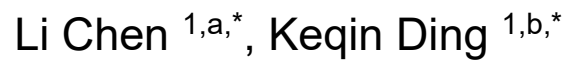 \\ ${ }^{1}$ China Special Equipment Inspection and Research Institute, Beijing 100029, China \\ aChenLi111528@163.com, b503718617@qq.com
}

\section{Keywords: Fatigue Life, Nonlinear Cumulative Damage, Lifting Equipment}

\begin{abstract}
The lifting equipment is applied more and more extensively with the fast development of economy. The fatigue and safety problems of lifting equipment in service are more and more prominent. This paper presents a nonlinear fatigue damage and life assessment approach for existing lifting equipment and the nonlinear effects of the fatigue damage accumulation due to random dynamic stress spectrum. The stress spectrum monitoring data was analyzed by the modified four peak-valley values fast rain-flow counting method for fatigue analysis. By considering nonlinear effects, the calculation for cumulative damage and prediction for fatigue life of the lifting equipment's hot region were calculated based on the nonlinear damage theory and the nominal stress method. The predicted fatigue damages are different when using the linear and nonlinear fatigue damage rules. According to the engineering application results, the real crack generation time is consistent with that of our estimation method, which demonstrates that our nonlinear prediction model and method for fatigue life are effective. The nonlinear damage theory is recommended for use in fatigue and damage prediction of lifting equipment in service.
\end{abstract}

\section{Introduction}

For many years, life prediction involving fatigue and damage assessment of key structure parts has been the research hot spot for experts and scholars, in many fields concerning national welfare and the people's daily life such as lifting equipment in service, engineering machinery, special equipment, etc. The safe problem of lifting equipment in service induced by fatigue and damage is becoming increasingly important.

A comprehensive review of many fatigue damage theories can be found elsewhere. Generally speaking, it can be divided into three categories, linear cumulative damage theory, double linear cumulative damage theory and nonlinear cumulative damage theory. Different fatigue damage theories have different accuracy and computational complexity. At present, Miner's linear cumulative damage theory is mainly used in engineering [1-2]. Although Miner's linear cumulative damage theory is simple and easy to use, the linear damage theory has been demonstrated to be unreliable. Studies [3] show that the median damage values to test specimens under certain loading conditions range from 0.15 to 1.06 . This is attributable to the fact that the relationship between physical damage and cycle ratio is not unique and varies from one stress level to another. Due to the complexity of fatigue damage evolution process and its mechanism, the nonlinear cumulative damage theory is needed [4-5].

In this paper, the fatigue life of large lifting equipment is studied based on the two-parameter rain flow fast counting program and the nonlinear cumulative damage theory.

\section{Fatigue life prediction method of lifting equipment}

Nominal stress method is a mature method for fatigue life estimation, and its prediction range is generally high cycle fatigue. The fatigue life of lifting equipment generally belongs to high cycle 
fatigue, so the nominal stress method will be used in this paper. The prediction steps are shown in figure 1 .



Figure 1 Fatigue Life Prediction Procedures

As shown in figure 1, obtaining S-N curves of key components is the basis for estimating fatigue life of components by nominal stress method. If the S-N curve of key components can be obtained directly through fatigue test of full-scale key components, the fatigue life of components can be accurately estimated. Generally speaking, the geometric shapes of key components are complex and diverse, so it is difficult to obtain the S-N curve directly from the fatigue test of full-scale key components.

The commonly used method is to estimate the S-N curve of key components according to the $\mathrm{S}-\mathrm{N}$ curve of standard sample materials.

The S-N curves of typical sample materials can be expressed as follows:

$$
N \sigma^{m}=C
$$

Or in logarithmic form:

$$
\lg (N)=\lg (C)-m \lg (\sigma)
$$

In order to estimate S-N curves of key components from S-N curves of standard sample materials, the influence of other factors should be taken into account. The expression of S-N curves of key components is as follows:

$$
N\left(K_{\sigma D} \sigma\right)^{m}=C
$$

Where $K_{\sigma D}$ is the correction coefficient of S-N curve of key components, which characterizes the influence of other factors (Including Fatigue notch coefficient $K_{f}$, Specimen Size coefficient $\varepsilon$, Surface Finishing coefficient $\beta$, Type of loading $C_{L}$ etc.) on fatigue properties of materials. $\mathrm{C}$ and $\mathrm{m}$ are material constants related to materials. Taking logarithm of formula (3) :

$$
\lg (N)=\lg (C)-m \lg \left(K_{\sigma D} \sigma\right)
$$




\section{Nonlinear fatigue cumulative damage theory}

The damage curve for another life level $\left(N_{i, f}\right)$ is then given by the power law damage equation defined as:

$$
D_{i}=\left(\frac{n_{i}}{N_{i, f}}\right)^{\left(\frac{N_{\mathrm{i}, f}}{N_{1, f}}\right)^{0.4}}
$$

For multiple life levels (e.g., $N_{1, f}<N_{2, f}<\cdots<N_{n, f}$ ), the damage curves (as shown in Figure 1) can be constructed expeditiously by letting the damage curve for the lowest life level be the reference life.

Nonlinear damage curves are shown in figure 2.

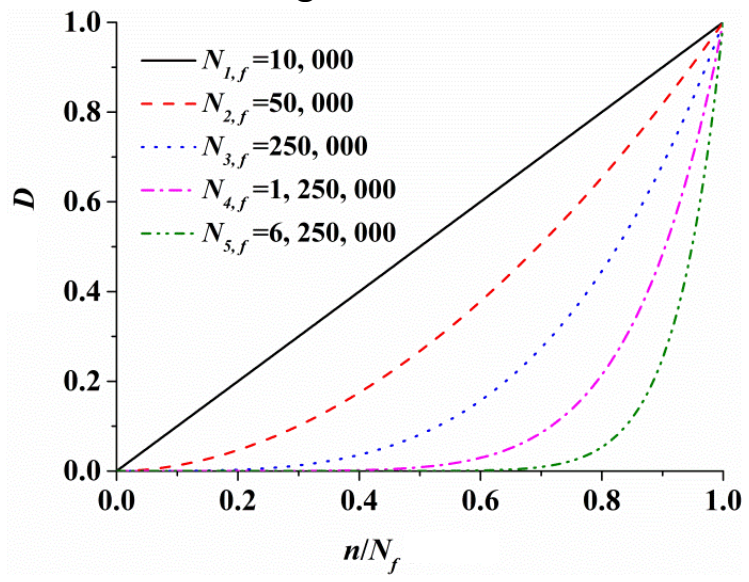

Figure 2 Nonlinear fatigue cumulative damage theory

\section{Data analysis based on rain-flow cycle counting method}

Based on the four peak valley value rain flow counting method [6-7], as shown in figure 3, the docking is processed from the peak or valley value in advance, and the final cycle count can be completed with only one rain flow count.
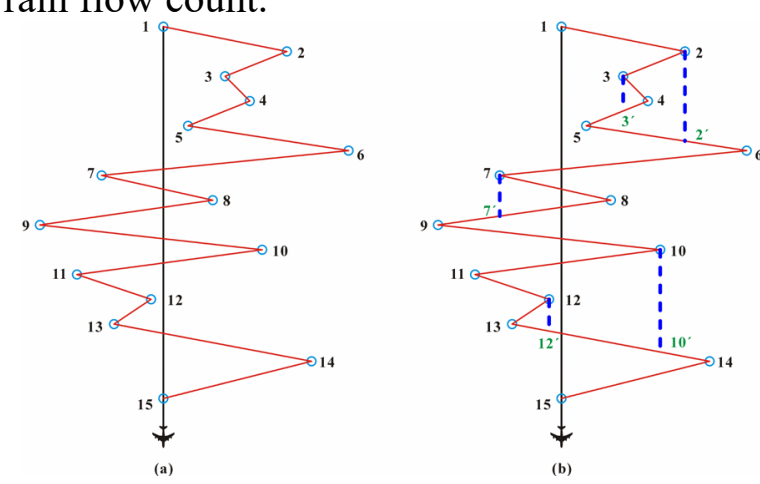

Figure 3 Rain-flow cycle counting method

The separation of the data to be processed and the data that needs to be kept can avoid repeated displacement of the data and greatly improve the processing efficiency. 


\section{Engineering application}

The monitoring position of metallurgy crane. The position of metallurgy crane plane stress state monitoring based on fiber Bragg grating sensor is shown in figure 4. The risk of crack initiation on position A2 is highest. So the random stress spectrum on position A2 is studied.
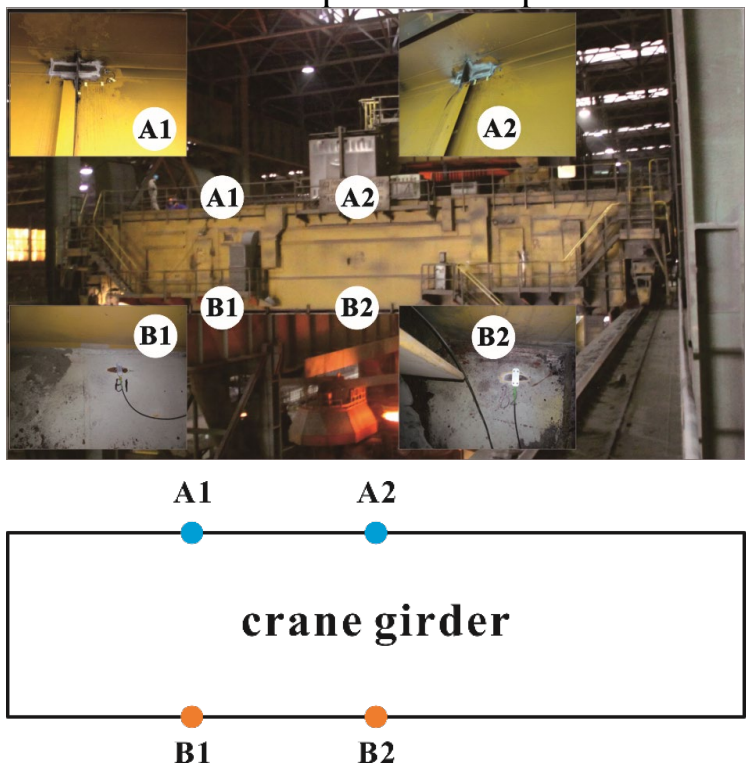

Figure 4 Fiber gratings position of monitoring point

Stress monitoring based on fiber Bragg grating sensor. As shown in figure 5, the main technical advantages of stress monitoring based on fiber Bragg grating sensor are as fellow:

(1) Fiber Bragg grating sensor can be multiplexed so that many sensors lie along a single fiber line. They are compatible with fiber optic data lines, which support huge necessary band width, which in turn support large number of sensors.

(2) Very micro thin. Therefore it can be made compatible with different composite material without changing mechanical properties.

(3) They can withstand high temperatures and pressure and are highly immune to electromagnetic interference.

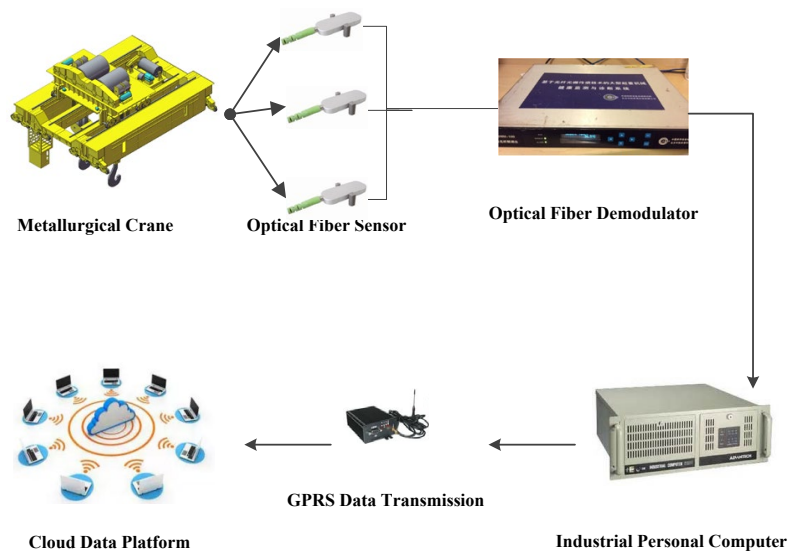

Figure 5 Structural Health Monitoring System for Lifting Equipment

Stress monitoring data based on structural health monitoring system are shown in figure 6. 


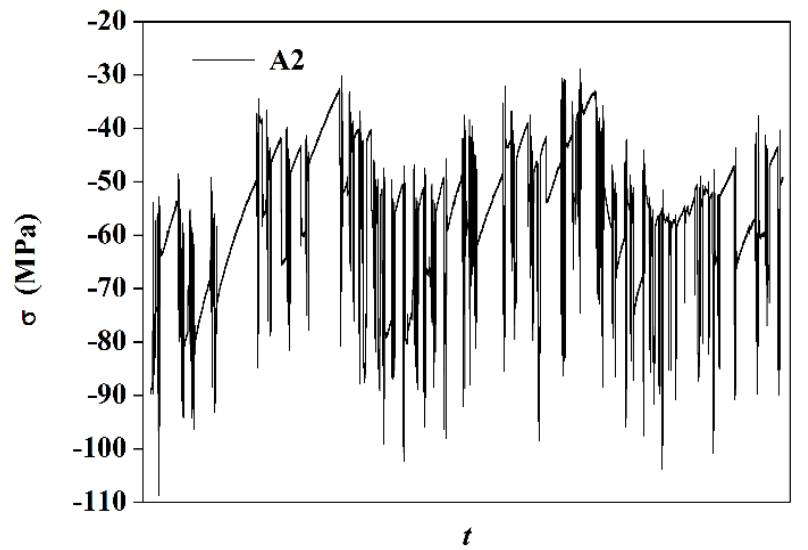

Figure 6 Structural Health Monitoring System for Lifting Equipment

The result based on nonlinear damage theory.

Table 1 Procedures for Fatigue Life Estimations Based on nonlinear damage theory

\begin{tabular}{ccccccccc}
\hline Blocks & $\mathrm{D}_{1}$ & $\mathrm{D}_{12}$ & $\mathrm{D}_{123}$ & $\mathrm{D}_{1234}$ & $\mathrm{D}_{1-5}$ & $\mathrm{D}_{1-6}$ & $\mathrm{D}_{1-7}$ & $\mathrm{D}_{1-8}$ \\
\hline 1 & 0.0111 & 0.0111 & 0.0118 & 0.0127 & 0.0138 & 0.0145 & 0.0151 & 0.0153 \\
2 & 0.0264 & 0.0264 & 0.0275 & 0.0289 & 0.0309 & 0.0322 & 0.0334 & 0.0339 \\
3 & 0.0450 & 0.0450 & 0.0463 & 0.0483 & 0.0510 & 0.0531 & 0.0549 & 0.0557 \\
4 & 0.0668 & 0.0668 & 0.0683 & 0.0708 & 0.0745 & 0.0773 & 0.0798 & 0.0809 \\
5 & 0.0920 & 0.0920 & 0.0938 & 0.0968 & 0.1013 & 0.1049 & 0.1083 & 0.1097 \\
6 & 0.1208 & 0.1208 & 0.1228 & 0.1263 & 0.1318 & 0.1363 & 0.1405 & 0.1424 \\
7 & 0.1535 & 0.1535 & 0.1557 & 0.1597 & 0.1662 & 0.1716 & 0.1768 & 0.1791 \\
8 & 0.1902 & 0.1902 & 0.1926 & 0.1972 & 0.2047 & 0.2111 & 0.2174 & 0.2202 \\
9 & 0.2313 & 0.2313 & 0.2339 & 0.2390 & 0.2476 & 0.2551 & 0.2626 & 0.2659 \\
10 & 0.2770 & 0.2770 & 0.2798 & 0.2855 & 0.2952 & 0.3039 & 0.3126 & 0.3166 \\
11 & 0.3277 & 0.3277 & 0.3307 & 0.3370 & 0.3479 & 0.3578 & 0.3679 & 0.3725 \\
12 & 0.3836 & 0.3836 & 0.3869 & 0.3937 & 0.4060 & 0.4172 & 0.4288 & 0.4341 \\
13 & 0.4452 & 0.4452 & 0.4486 & 0.4561 & 0.4697 & 0.4823 & 0.4956 & 0.5017 \\
14 & 0.5127 & 0.5127 & 0.5164 & 0.5246 & 0.5396 & 0.5537 & 0.5686 & 0.5756 \\
15 & 0.5867 & 0.5867 & 0.5906 & 0.5994 & 0.6159 & 0.6316 & 0.6484 & 0.6563 \\
16 & 0.6674 & 0.6674 & 0.6715 & 0.6810 & 0.6990 & 0.7165 & 0.7353 & 0.7442 \\
17 & 0.7553 & 0.7553 & 0.7596 & 0.7698 & 0.7895 & 0.8088 & 0.8298 & 0.8398 \\
18 & 0.8508 & 0.8508 & 0.8554 & 0.8663 & 0.8878 & 0.9090 & 0.9323 & 0.9434 \\
$\mathbf{1 9}$ & 0.9545 & 0.9545 & 0.9593 & 0.9709 & 0.9942 & $\mathbf{1 . 0 1 7 4}$ & $\mathbf{1 . 0 4 3 2}$ & $\mathbf{1 . 0 5 5 6}$ \\
\hline
\end{tabular}

According to table 1, the fatigue life of the hot spot area are as follows:

Fatigue Life $=19($ Years $)$

The metallurgical crane was put into operation in 1997. 20 years later, cracks were detected in hot spot area A2 in 2017. The real crack generation time (20 years) is consistent with that of our estimation (19 years), which demonstrates that our prediction model and method for fatigue life are effective and give a method for the evaluation of the lifting equipment's fatigue life. 


\section{Conclusion}

In this paper, the nominal stress method is used as the basic method of fatigue life prediction. First, the monitoring stress spectrum is processed by two-parameter rain-flow counting method. Then, the fatigue cumulative damage caused by monitoring stress spectrum is calculated based on the nonlinear fatigue cumulative damage theory. Finally, the fatigue life is analyzed and predicted.

According to the engineering application results, the real crack generation time is consistent with that of our estimation, which demonstrates that our prediction model and method for fatigue life are effective and give a method for the evaluation of the lifting equipment's fatigue life.

\section{Acknowledgments}

This research was supported by National key technologies Research \& Development program through 2017YFC0805100.

\section{References}

[1] Nykänen T, H Mettänen, T Björk, A Ahola. (2016) Fatigue assessment of welded joints under variable amplitude loading using a novel notch stress approach. International Journal of Fatigue, 101: S0142112316304315. https://doi.org/10.1016/j.ijfatigue.2016.12.031

[2] Durodola J F, N Li, S Ramachandra, A N Thite. (2017) A pattern recognition artificial neural network method for random fatigue loading life prediction. International Journal of Fatigue, 99: 55-67. https://doi.org/10.1016/j.ijfatigue.2017.02.003

[3] Lee Y L, M W Lu, R C Segar, C D Welch, R J Rudy. (1999) Reliability-based cumulative fatigue damage assessment in crack initiation. International Journal of Materials \& Product Technology, 14: 1-16. https://doi.org/10.1504/IJMPT.1999.036257

[4] Shang D G, W X Yao. (1999) A nonlinear damage cumulative model for uniaxial fatigue. International Journal of Fatigue, 21: 187-194. https://doi.org/10.1016/S0142-1123(98)00069-3

[5] Wu W F, T H Huang. (1991) A statistical nonlinear cumulative damage rule and fatigue life prediction under random loading. International Journal of Pressure Vessels \& Piping, 47: 1-16. https://doi.org/10.1016/0308-0161(91)90083-E

[6] Gupta S, I Rychlik. (2007) Rain-flow fatigue damage due to nonlinear combination of vector Gaussian loads. Probabilistic Engineering Mechanics, 22: 231-249. https://doi.org/10.1016/j.probengmech.2007.04.003

[7] Rychlik I, S Gupta. (2007) Rain-flow fatigue damage for transformed gaussian loads. International Journal of Fatigue, 29: 406-420. https://doi.org/10.1016/j.ijfatigue.2006.05.006 